\title{
Selection in touch: Negative priming with tactile stimuli
}

\author{
Christian Frings and Regine Bader \\ Saarland University, Saarbrücken, Germany \\ AND \\ Charles Spence \\ University of Oxford, Oxford, England
}

\begin{abstract}
Abundant experimental evidence has demonstrated attentional selection within the visual modality. Furthermore, the consensus view is currently that two processes contribute to selection: the amplification of the target stimuli and the ignoring (or suppression) of any distractor information. However, at present it is less clear how selection is achieved within the tactile modality. In the present study, we analyze the aftereffects of ignoring tactile distractors, in a novel tactile variant of the negative priming paradigm. In the typical negative priming paradigm, repeating an ignored distractor stimulus as the target on the following trial usually leads to a cost in terms of reaction times (RTs) or error rates, thereby indicating that selection is achieved in part by the ignoring of distractors. In two experiments, we observed significant RT costs when a previously ignored vibrotactile stimulus constituted the target in the next trial. This result shows that tactile selection is in part achieved by active ignoring of distractor representations, as has been shown previously in both the visual and auditory modalities.
\end{abstract}

At every waking moment, a wealth of information from the various sensory systems is processed by our brains. The efficient control of action, however, requires that only certain features of particular stimulus representations should be fully processed and subsequently used to guide actions (see, e.g., Allport, 1987). Selective attention helps to both find and selectively process relevant information, at the expense of irrelevant information. In fact, selection is considered to represent one of the principal functions of attention (see Pashler, 1998). By and large, it has been assumed that selection is achieved by supporting the processing of relevant information by facilitating the processing of relevant stimuli and/or by inhibiting or ignoring the processing of irrelevant stimuli.

Abundant evidence for unimodal selection in the visual and auditory modalities has now been collected (see, e.g., Allport, 1993; Johnston \& Dark, 1986). In addition, there is growing interest in the representation and selection of multisensory inputs (see, e.g., chapters in Calvert, Spence, \& Stein, 2004, and Spence \& Driver, 2004, as well as Dell'Acqua, Turatto, \& Jolicœur, 2001; Marks, 2004). To date, very few studies have attempted to analyze unimodal selection within the tactile modality. One paradigm that has been argued to tap directly into the ignoring of distractors in the visual modality - namely, the negative priming (NP) paradigm - has not, as yet, been tested with tactile stimuli. Thus, the present study focuses on the ignoring of tactile distractors when tactile targets must be selected from among these distractors by utilizing the NP paradigm with tactile stimuli.

\section{Tactile Selection}

Early studies of tactile selection typically analyzed participants' detection performance when two tactile stimuli were presented simultaneously. Detection performance has been shown to be affected by the separation of the target and the distractor on the skin surface (see, e.g., Craig, 1974; Uttal, 1960). Researchers have also shown that the similarity between the target and distractor stimuli can modulate tactile discrimination performance as well (see, e.g., Driver \& Grossenbacher, 1996; Evans \& Craig, 1991; Soto-Faraco, Ronald, \& Spence, 2004). Target responses have tended to be faster and more accurate when distractors were identical to the target, as compared with those trials in which the targets and distractors differed. However, it has remained unclear whether target selection in those studies was impaired by the masking provided by patterns of distractors that were different from a target (early selection) or whether the distractors elicited a response incompatible with the target response (late selection). Using a paradigm in which four stimuli were mapped onto two possible responses, Craig (1995; Craig \& Evans, 1995) demonstrated that target selection was significantly impaired by the presentation of response-incompatible as compared with response-compatible distractors, although in both conditions the identity of the distractors was al- 
ways different from that of the target. Thus, the distractor information interfered with selection of the target information at the level of response competition, thereby suggesting that distractors are fully analyzed and processed up to the level of evoking responses; yet, Craig (2000) also demonstrated that response-neutral distractors interfere with target responding because of temporal masking. ${ }^{1}$

However, one question that has not as yet received an empirical answer concerns the fate of the distractor representation. Is it actively ignored, or is tactile selection mainly achieved by facilitating the representation of the target stimulus? The present study attempted to answer this question using a novel tactile NP paradigm. It should be noted that in some recent studies, tactile selection was analyzed when the target stimulus had to be selected after the presentation of a cue (see, e.g., Spence, Nicholls, Gillespie, \& Driver, 1998) or when the target stimulus had to be selected from a stream of rapidly presented stimuli (Dell'Acqua, Jolicœur, Sessa, \& Turatto, 2006; Hillstrom, Shapiro, \& Spence, 2002; Soto-Faraco et al., 2002); in some of these studies, evidence for target facilitation was observed. For example, when participants responded rapidly to a target following a cue at the same location, reaction time (RT) benefits were observed. These benefits were explained in terms of target facilitation.

However, here we focus on the fate of distractor representations in more traditional selection situations. Two differences from other studies should be noted. First, in our task, a target had to be selected from among responseevoking distractors by utilizing a tactile variant of the standard NP task. Thus, the target was presented together with the distractor, and the analysis focused on whether reactions to the distractor pattern were impaired when the distractors were ignored. Second, we used complex tactile patterns instead of the short pulses usually used in studies of tactile attention (Driver \& Grossenbacher, 1996; Soto-Faraco et al., 2004). We did this because, in real life, tactile information is generally more complex than a brief pulse. For example, when using tactile inputs for regulating fine motor skills, the relevant tactile patterns are facilitated, whereas all other tactile patterns experienced at the same time are ignored. Thus, here we analyzed, for the first time, realistic and complex selection in touch.

\section{The NP Paradigm}

In the typical NP task, the stimuli are presented in two consecutive displays: the first display known as the prime display, the second as the probe display. In each one, the target and distractor stimuli (e.g., typically words or pictures) are presented close together in time, and the participants are instructed to ignore one (the distractor) while attending and responding to the other (the target). Typically, participants' reactions to the target in the second display are slowed if this stimulus constituted the distractor on the preceding display, the so-called NP effect (see, e.g., Fox, 1995, and May, Kane, \& Hasher, 1995, for reviews). This slowing of participants' responses is assumed to reflect an aftereffect of ignoring the distracting prime item. Thus, there appears to be a general consensus in the literature that NP taps into selective control mechanisms (see Tipper, 2001). However, the exact nature of these control processes is still being debated. A coarse-grained taxonomy of theories of NP differentiates between inhibition-based (Houghton \& Tipper, 1994; Tipper, 1985) and retrievalbased (Milliken, Joordens, Merikle, \& Seiffert, 1998; Neill, 1997; Rothermund, Wentura, \& De Houwer, 2005) accounts. According to the inhibition theory, the representation of the distractor stimulus is thought to be actively suppressed by mechanisms of selective attention during the processing of the prime episode, an inhibition that persists until the presentation of the next display. Thus, when the ignored distractor from the previous trial becomes the target in the current trial (the ignored-repetition [IR] condition), the recently inhibited representation now has to be activated in order for the participant to respond correctly, so that NP occurs. It should be noted, however, that Joordens, Betancourt, and Spalek (2006) recently showed that even attended distractors cause NP when participants selectively responded to the prime target; that is, there is some debate whether NP reflects selective attention or selective responding. By contrast, according to retrieval theories, NP is caused by the fact that perceiving a target activates memory traces associated with that particular stimulus. In the IR condition, the last memory trace of the current target stimulus may contain information like "distractor" or "do not respond," and this information interferes with the execution of rapid and accurate responses to the current target. Both accounts are well supported by the available empirical data, which has led several authors to conclude that both inhibitory mechanisms and retrieval processes most likely contribute to NP (see, e.g., Kane, May, Hasher, Rahhal, \& Stoltzfus, 1997; Tipper, 2001).

In summary, although theoretical debate concerning the causes of NP continues, there is at least a consensus view that NP is caused by selectively responding to the prime target. Therefore, if NP can be found using tactile stimuli, this would suggest that tactile selection probably involves the ignoring of the distractor stimulus, just as has been demonstrated for the case of intramodal visual selection. Thus, the visual NP paradigm was adopted for use in the tactile modality in two separate experiments. In each trial, the participants had to try to identify the vibrotactile target stimulus while simultaneously ignoring the distractor vibration. Slower responses were expected in those trials in which a vibrotactile stimulus that had been ignored in the previous trial constituted the target in the current trial.

\section{EXPERIMENT 1}

In this experiment, four vibrotactile patterns were presented in the context of an identification task. One target vibrotactile stimulus and one distractor vibrotactile stimulus were presented simultaneously in each trial until participants responded to the target stimulus. The location of the target was unpredictable and was cued by the color of the display at the start of each trial. A learning phase was presented prior to the experimental phase in order to reduce participants' error rates. 


\section{Method}

Participants. Seventeen students from Saarland University took part in this experiment for course credit. Their median age was 22 years (range 20-31 years). All had a normal sense of touch and normal or corrected-to-normal vision by self-report. All of the participants were naive as to the purpose of the experiment.

Design. The experiment consisted of a 2 (probe target position: left vs. right) $\times 2$ (probe response hand: left vs. right) $\times 2$ (priming condition: ignored repetition vs. control) within-participants design. Prime response hand and prime target position were also varied orthogonally with respect to the other factors. NP effects were computed as the difference in performance between control $(\mathrm{C})$ and IR trials.

Apparatus and Materials. The experiment was conducted on a standard PC with a 17-in. CRT screen. Vibrotactile stimuli were delivered by means of two skin transducers (Model VBW32, Audiological Engineering Corp.). Each transducer had a mass of $6.5 \mathrm{~g}$ (including the Velcro strip used to fasten it to the participant's hand) and was $2.54 \times 1.85 \times 1.06 \mathrm{~cm}$ in size. The transient response of each transducer was measured at $5 \mathrm{msec}$ (attack and decay). The amplitude range extended $50 \mathrm{~dB}$ above the sensory threshold. E-Prime (Version 1.1) was used to conduct the experiment and to control the presentation of the sound files. A Sony hi-fi system was used to amplify the sounds. Instead of normal loudspeakers, however, the skin transducers were connected to the amplifier. Thus, the sound files were used to generate vibrotactile stimuli presented via the transducers. Four tones with different rhythms were created as the vibrotactile stimuli, using the freeware program Audacity (Version 1.2.6). The stimuli were presented repeatedly until the participant responded or until 5,000 msec had elapsed since the beginning of the trial. One stimulus consisted of a single longer pulse (340 msec) followed by three shorter pulses (180 msec each). Two pauses of $60 \mathrm{msec}$ each separated the pulses, one after the longer pulse and one after the last of the three short pulses. The duration of the whole sequence was $1,000 \mathrm{msec}$. Another stimulus comprised four pulses of equal length (192 msec each) and intensity, with each pulse being followed by a $58-\mathrm{msec}$ pause. The third stimulus was composed of one short pulse $(110 \mathrm{msec})$ followed by an 890 -msec pause, and the fourth stimulus simply consisted of a continuous vibration presented until the participant reacted or until 5,000 msec had elapsed.

Procedure. Participants were tested individually. Each participant sat $60 \mathrm{~cm}$ in front of the PC, with the skin transducers fixed onto his or her palms. A transducer was placed on the side of each of the participant's palms (see the middle panel of Figure 1). Participants had to identify the target vibrations by pressing assigned keys on a QWERTY keyboard in front of them. To limit the noise made by the transducers touching the table, armrests were provided on which the participants put their wrists. Instructions were given on the screen and summarized by the experimenter at the beginning of each phase of the experiment. Each participant placed the left middle finger on the "c" key, the left index finger on the " $v$ " key, the right index finger on the " $\mathrm{n}$ " key, and the right middle finger on the " $\mathrm{m}$ " key. White noise was presented over headphones to mask the sounds made by the operation of the vibrotactile stimulators (see the left panel of Figure 1 for a bird's-eye view of the test arrangement).

The experimental session comprised four training phases, in which the assignment of vibrotactile stimuli to specific keys on the keyboard was learned, and an experimental phase in which participants had to perform the NP task itself. In the experimental phase, each trial consisted of the following events (see the right panel of Figure 1): The participant initiated each trial by pressing the space bar. Next, the prime was presented. This comprised two stimuli (i.e., the target vibration and the distractor vibration). Both stimuli were presented simultaneously until the participant responded. The participant was then instructed to identify the target and to ignore the vibrotactile distractor stimulus, and to respond as rapidly and accurately as possible. Simultaneous with the onset of the stimuli, the display changed color; the screen also remained colored until the participant responded. A red screen indicated that the target stimulus would be presented to the right hand, whereas a blue screen indicated that the target would be presented to the left hand. The Velcro strips with which the tactile stimulators were taped to the palm were colored in a congruent manner (i.e., the Velcro strip on the participant's right hand was red, whereas the Velcro strip on the left hand was blue). Thereafter, a white screen was shown for 1,000 msec, followed by the probe. The probe also consisted of two stimuli, one the target and the other the distractor. Again, the participant had to identify the target vibration that was indicated by the color of the screen.

All possible combinations of each probe with two different stimuli were constructed by orthogonally varying the positions of the stimuli, resulting in 24 different probes. Since we were only interested in IR and C conditions, we built all possible primes for each probe that would transfer this prime-probe sequence into an IR or a $\mathrm{C}$ trial (four IR primes and four $\mathrm{C}$ primes for each probe). This design resulted in a total of 192 experimental trials (96 IR and 96 C). In an IR trial, the prime distractor was repeated as the probe target, whereas in a $\mathrm{C}$ trial all four stimuli were different. The trials were presented in a pseudorandomized order. Twelve practice trials (which were not analyzed) were presented prior to the experimental trials.

At the start of the experiment, the participants had to learn the assignments of the vibrotactile stimuli to the response keys. In each trial of Learning Phase 1 , one of the vibrations was randomly presented to

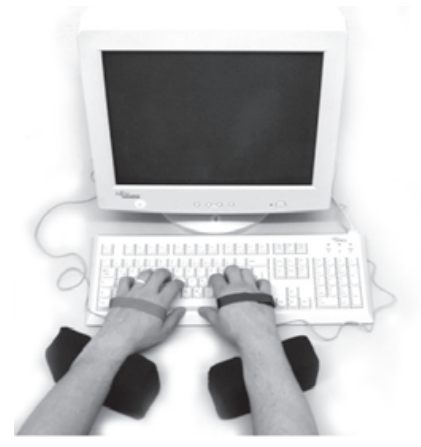

Bird's-eye view of the test arrangement
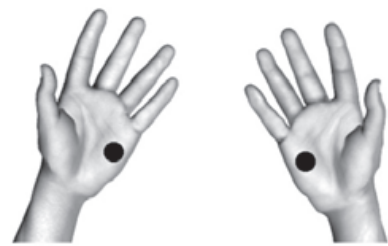

The skin transducers were taped on the palms of a participant's hands.

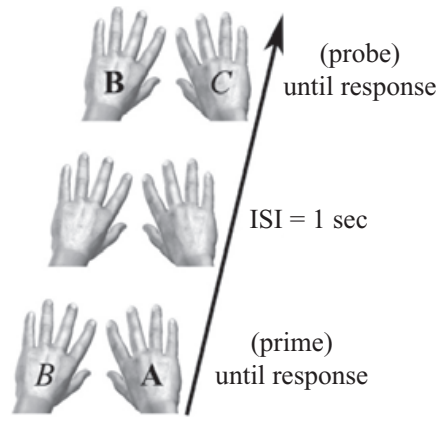

An example of an IR trial (The letters A, B, and C stand for the different vibrations.

Bold letters $=$ target; italic letters $=$ distractor. $)$

Figure 1. Procedural details of the test arrangement for the tactile negative priming task (see the text for details). 
either a participant's left or right hand. Simultaneously, an image was displayed on the screen that showed a photograph of one left and one right hand. One finger in this image was colored green, indicating the corresponding response finger for this vibration. The background of this image was white, and a trial ended when the participant responded. Each vibration was presented three times to each hand (i.e., there were 24 trials in total). We used photographs to achieve a direct mapping of responses to vibrotactile patterns, thereby minimizing possible strategies of the participants (e.g., verbal recoding of the tactile patterns). In Learning Phase 2, the participants were given practice in attending to a specific target hand. The procedure was the same as in Learning Phase 1, except that the background of the display was now either red or blue. When the display was red, participants sensed the vibration on their right hand, and when it was blue, they sensed it on their left hand. This phase of the experiment also consisted of 24 trials. In Learning Phase 3, we tested whether the participants had learned the correct stimulus-response mapping between the vibrotactile stimuli and the fingers they had to respond with. Once again, the color of the screen (red or blue) indicated the hand on which the target vibration would be presented. The participants were instructed to try to identify the vibration and respond with the appropriate finger. This time, however, the photograph of the two hands that indicated the correct finger was no longer displayed. Feedback concerning the correctness of participants' responses was given after each trial. The four vibrations were presented repeatedly in a random order until participants were able to correctly identify each of the vibrations five times in a row (on average, the participants needed $10 \mathrm{~min}$ to fulfill this learning criterion). Finally, in Learning Phase 4 , the participants were presented with 48 trials containing both targets and distractors. These trials were exactly the same as the experimental trials, except that the participants were still given feedback about the correctness of their answers. The length of all four learning phases was about $20-25 \mathrm{~min}$ in total.

\section{Results}

Only correct probe RTs were analyzed (the error rate on the probe displays was $22.9 \%$ ). Moreover, RTs had to be longer than $350 \mathrm{msec}$ and lower than the Tukey (1977) outlier criterion (i.e., 1.5 interquartile ranges above the third quartile; this criterion was $4,100 \mathrm{msec}$, and $3.5 \%$ of the trials were discarded as outliers). With respect to these criteria, $26.4 \%$ of trials were discarded. The mean RTs and error rates are highlighted in Table 1.

Reaction times. Correct probe RTs were subjected to a 2 (priming condition: IR vs. C) $\times 2$ (probe response hand: left vs. right) $\times 2$ (probe target position: left vs. right) MANOVA. A significant main effect of priming condition emerged $\left[F(1,16)=16.96, p=.001, \eta^{2}=.52\right]$, indicating a significant NP effect (i.e., slowed RTs to probe target vibrations that had been presented as distractor vibrations in the prime; $M=-85 \mathrm{msec}, S D=85 \mathrm{msec}, d z=1.0$; 15 of the 17 participants showed NP, $p=.002$ in a sign test). The main effects for probe response hand and probe target position were also significant $[F(1,16)=51.88$, $p<.001, \eta^{2}=.76$, and $F(1,16)=6.84, p=.02, \eta^{2}=$ .30 , respectively], showing that the participants generally responded more rapidly with the right hand and when targets were presented to the right hand. In addition, there was a significant interaction between probe response hand and probe target position $\left[F(1,16)=5.48, p=.03, \eta^{2}=\right.$ .26], indicating a compatibility effect of target position and response hand (i.e., the participants responded more rapidly when the target was presented to the hand that executed the response). None of the other interactions were significant (all $F_{\mathrm{S}}<1.33$, all $p \mathrm{~s}>.26$ ). ${ }^{2}$

Error rates. We conducted the same MANOVA on the error data. However, the only significant term was a main effect of probe response hand $[F(1,16)=28.17, p<.001$, $\left.\eta^{2}=.64\right]$, indicating that the participants made fewer errors when responding with the right hand. None of the other terms in the analysis were significant (all $F_{\mathrm{S}}<2.8$, all $p \mathrm{~s}>.11)$.

\section{Discussion}

The results of Experiment 1 demonstrate for the first time that NP occurs in the tactile modality. This result supports the claim that the representation of a tactile distractor is fully processed and then inhibited, or tagged, with some kind of "do not respond" instruction. If such a stimulus then becomes the target in the next trial, the RT increases. This result suggests that tactile selection is also achieved by ignoring the distractor, not only by facilitating the representation of the target stimulus. We will discuss the implications of this finding in the General Discussion.

A significant interaction was also observed between the response hand and target location factors; that is, we observed a stimulus-response compatibility, or Simon, effect (see Röder, Kusmierek, Spence, \& Schicke, 2007; Simon, 1990), indicating that the participants responded more rapidly when the target was presented to the hand with which they responded on that trial, although response location was irrelevant for the identification task per se.

However, it should be noted that Experiment 1 differed somewhat from previous studies of NP in vision, in that the feature that specified the target (here, location) was not de-

Table 1

Mean Reaction Times (RTs, in Milliseconds) and Percentages of Errors As a Function of Priming Condition, Probe Response Hand, and Probe Target Position in Experiment 1

\begin{tabular}{|c|c|c|c|c|c|c|c|c|}
\hline \multirow[b]{2}{*}{ Priming Condition } & \multicolumn{4}{|c|}{ Probe Target Left } & \multicolumn{4}{|c|}{ Probe Target Right } \\
\hline & RT & $S D$ & $\%$ & $S D$ & RT & $S D$ & $\%$ & $S D$ \\
\hline \multicolumn{9}{|c|}{ Response Hand Left } \\
\hline Ignored repetition & 2,099 & 284 & 32.8 & 22.3 & 2,285 & 416 & 35.0 & 20.3 \\
\hline Control & 2,053 & 354 & 32.3 & 21.8 & 2,276 & 410 & 38.5 & 24.1 \\
\hline \multicolumn{9}{|c|}{ Response Hand Right } \\
\hline Ignored repetition & 1,928 & 473 & 13.4 & 19.5 & 1,919 & 268 & 9.6 & 11.2 \\
\hline Control & 1,802 & 228 & 12.0 & 16.9 & 1,760 & 217 & 9.3 & 9.5 \\
\hline
\end{tabular}


fined until both stimuli were presented. By contrast, in visual NP tasks, the target stimuli have most commonly been identified by color, with the target color remaining constant throughout the experiment (or at least throughout a block of trials). One study even found diminished NP effects when the target color changed between prime and probe displays (see MacLeod, Chiappe, \& Fox, 2002). In the present study, we varied the feature that specified the target vibration (i.e., the display color indicating the target hand) unpredictably from trial to trial. In this regard, it should be noted that Soto-Faraco et al. (2004) found a significant increase in the magnitude of the interference from irrelevant distractors in a tactile selection task when they made the target location (i.e., the target hand) unpredictable. In Experiment 2, we therefore tested whether tactile NP would also be found when target location was predictable.

\section{EXPERIMENT 2}

This experiment constitutes a replication of Experiment 1 , with the difference that target location was varied on a block-by-block basis; selection in this experiment was therefore completely analogous to selection in visual NP tasks.

\section{Method}

Participants. Twenty new students from Saarland University took part for course credit. Their median age was 22 years (range 20-49 years). All of the participants had normal touch and normal or corrected-to-normal vision by self-report.

Apparatus, Materials, Design, and Procedure. Experiment 2 was an exact replication of Experiment 1, with the following exceptions: (1) The positions of the vibrotactile targets were varied in a blockwise manner; that is, in the first block (consisting of 96 trials), all of the targets were presented to the right hand, whereas in the second (also consisting of 96 trials), all of the targets were presented to the left hand (or vice versa, with the block sequence counterbalanced across participants). (2) Before each block, Learning Phase 4 and a warm-up phase of 12 trials were re-presented to the participants. The targets in these practice trials appeared only at the target hand of the following experimental trials.

\section{Results}

Only correct probe RTs were analyzed (the error rate on probe displays was $12.7 \%$ ). Moreover, RTs had to be longer than $350 \mathrm{msec}$ and below the Tukey (1977) outlier criterion (in the present experiment, this criterion was $3,391 \mathrm{msec}$, leading to $4.9 \%$ of the trials being discarded).
The exclusions of outliers and error probes resulted in the elimination of $17.6 \%$ of the trials. The mean RTs and error rates are shown in Table 2.

Reaction times. The block sequence (i.e., whether the target was presented to the left or right hand first) did not interact significantly with the priming condition $(p=.73)$ and hence was dropped from the analysis. As in Experiment 1 , correct probe RTs were subjected to a 2 (priming condition: IR vs. C) $\times 2$ (probe response hand: left vs. right) $\times 2$ (probe target position: left vs. right) MANOVA. Once again, a significant main effect of the priming condition was observed $\left[F(1,19)=11.84, p=.003, \eta^{2}=\right.$ .38 ], indicating a significant NP effect $(M=-73 \mathrm{msec}$, $S D=94 \mathrm{msec}, d z=0.77 ; 15$ of the 20 participants tested showed NP, $p=.04$ in a sign test). The main effect of probe response hand was also significant $[F(1,19)=17.53, p=$ $\left..001, \eta^{2}=.48\right]$, showing faster responses with the right hand. The only other significant effect was a marginally significant three-way interaction between priming condition, response hand, and target position $[F(1,19)=4.23$, $\left.p=.05, \eta^{2}=.18\right]$. This interaction term shows that NP was more reliable in those trials in which the target position and response hand were compatible. No other effects were significant (all $F \mathrm{~s}<2.84$, all $p \mathrm{~s}>.10$ ).

Error rates. A similar MANOVA was conducted on the error data. As in Experiment 1, the only significant term was the main effect of probe response hand $[F(1,19)=$ $26.46, p<.001, \eta^{2}=.58$ ], indicating that the participants made fewer mistakes when they responded with the right hand. None of the other effects were significant (all $F \mathrm{~s}<$ 2.8 , all $p \mathrm{~s}>.11){ }^{3}$

Comparison of the results of Experiments 1 and 2. RTs were, on average, significantly faster, and error rates significantly lower, in Experiment 2 than in Experiment 1 (both $p \mathrm{~s}<.01$ ), thus indicating that target selection was easier for participants when the position of the target was predictable (see Soto-Faraco et al., 2004). We also compared the NP effects from both experiments. However, although the tactile NP effect was numerically somewhat larger in Experiment $1(M=85 \mathrm{msec}, d z=1.0)$ than in Experiment $2(M=73, d z=0.77)$, this difference was far from significant $[t(35)=0.42, p=.68]$.

\section{Discussion}

The overall decrease in RTs and the reduction in error rates in Experiment 2 as compared with Experiment 1 ap-

Table 2

Mean Reaction Times (RTs, in Milliseconds) and Percentages of Errors As a Function of Priming Condition, Probe Response Hand, and Probe Target Position in Experiment 2

\begin{tabular}{|c|c|c|c|c|c|c|c|c|}
\hline \multirow[b]{2}{*}{ Priming Condition } & \multicolumn{4}{|c|}{ Probe Target Left } & \multicolumn{4}{|c|}{ Probe Target Right } \\
\hline & RT & $S D$ & $\%$ & $S D$ & RT & $S D$ & $\%$ & $S D$ \\
\hline \multicolumn{9}{|c|}{ Response Hand Left } \\
\hline Ignored repetition & 1,769 & 401 & 17.3 & 14.8 & 1,756 & 376 & 20.2 & 18.9 \\
\hline Control & 1,671 & 357 & 15.4 & 13.2 & 1,783 & 378 & 20.4 & 16.9 \\
\hline \multicolumn{9}{|c|}{ Response Hand Right } \\
\hline Ignored repetition & 1,580 & 292 & 8.5 & 8.4 & 1,560 & 306 & 6.0 & 7.6 \\
\hline Control & 1,490 & 261 & 7.1 & 5.9 & 1,431 & 272 & 6.5 & 7.0 \\
\hline
\end{tabular}


pear to suggest that the participants found target selection easier in our second experiment. This presumably occurred because they were able to selectively focus attention on the target hand in advance of stimulus presentation. Nevertheless, the key point to note is that a significant tactile NP effect was still observed, even when selection became easier because target position was made predictable in advance. We should note, however, that the NP effect reported in Experiment 2 was numerically somewhat smaller than that reported in Experiment 1 (although this difference failed to reach statistical significance), and furthermore that the effect size dropped from $d z=1.0$ to 0.77 . This trend toward a reduction in the magnitude of the NP effect is in line with the results of previous studies of visual NP, in which the difficulty of selection was shown to modulate the NP effect (e.g., Frings \& Wühr, 2007; Houghton, Tipper, Weaver, \& Shore, 1996; Ruthruff \& Miller, 1995) — with NP increasing as selection became more difficult. Moreover, studies of selection within the tactile modality have also revealed a decrease in tactile interference effects when the target hand was made predictable in advance (Soto-Faraco et al., 2004).

The marginally significant three-way interaction between priming condition, probe response hand, and probe target location indicates that vibrotactile NP was primarily found in the trials in which response and location were compatible (see Table 2). This result may have been caused by one of two processes: First, it may be that, in general, response-incompatible trials yield more imprecise measurements of NP simply because the response incompatibility adds some noise to the measurement of NP. Second, in this experiment, when the target location was fixed, each response-compatible IR probe followed a responseincompatible prime with a probability of $67 \%$, and vice versa. Thus, it might be assumed that participants reacted more accurately after a response-incompatible trial, and hence the measurement was more precise in the following IR probe trial. This theory may also help explain why this interaction was not observed in Experiment 1, in which the target location varied from trial to trial.

\section{GENERAL DISCUSSION}

The results of the two experiments reported in this study provide the first empirical evidence that NP can influence the processing of vibrotactile stimuli. This result shows that the selection of task-relevant tactile information is achieved by at least two separable processes - namely, the facilitation of the target representation, as shown in previous studies (see Spence, Lloyd, McGlone, Nicholls, \& Driver, 2000), and actively ignoring the distractor representations. In this respect, selection in the tactile modality parallels selection in the visual modality (Neumann, 1996; Tipper, 2001). Moreover, we analyzed here selection with complex vibrotactile patterns. So far, little is known about how such stimuli are processed, albeit that in everyday life most tactile stimuli encountered are complex patterns.

As stated in the introduction, in the literature an ongoing debate concerns whether NP reflects the retrieval or inhibition of the prime distractor. It is important to note that both approaches to NP are consistent with the data presented here. According to inhibition theory, during the presentation of the prime, the distractor representation is actively inhibited, and this inhibition persists until presentation of the probe. Thus, in an IR trial, the probe target representation is still inhibited as it is presented, and selection of the probe target rather than a noninhibited distractor is thereby prolonged, resulting in NP. On the other hand, according to retrieval theories, the distractor representation is encoded with a "do not respond" tag during the presentation of the prime, and this tag is retrieved in the following probe (in the case of an IR trial); the retrieved response instruction interferes with the demanded probe response, and thus NP occurs.

However, it is important to note that the question of whether NP reflects retrieval or inhibition was not the focus of the two experiments reported here. Instead, we addressed the question of how selection within the tactile modality is actually achieved. Previous studies on tactile selection analyzed whether interference effects from distractors were due to masking or response competition. Craig (1995) showed that tactile distractors are processed to a level at which responses are programmed, and therefore can cause interference at the level of response competition. However, it has previously been unclear what actually happens to the representation of tactile distractors. Here, we have presented the first empirical evidence that ignoring a tactile distractor in a situation requiring selection can influence a person's next encounter with this stimulus; that is, not only is the representation of the tactile distractor processed to the level at which it evokes a response, thus producing response competition, but the distractor representation is actively ignored as well, in order to further support target selection. Thus, repeating a previously ignored vibrotactile stimulus as the target on the next trial produces RT costs, showing the aftereffects of the ignoring of the prime distractor.

It should also be noted that the NP effects and effect sizes observed here in tactile selection tasks were very large $(d z=1.0$ in Experiment 1 and $d z=0.77$ in Experiment 2). For example, in NP experiments from our laboratory using visual selection tasks, the average NP effect size is between $d z=0.20$ to $d z=0.50$ (e.g., Frings, 2006; Frings \& Wentura, 2006a, 2006b; Frings \& Wühr, 2007). In contrast, studies using auditory stimuli have produced quite large NP effect sizes, from $d z=0.85$ to $d z=1.08$ (Mayr \& Buchner, 2006). The reason for these differences in NP, however, might not be a modality-specific effect. In fact, the amount of NP seems to be a function of the difficulty of selection (see Frings \& Wühr, 2007; Ruthruff \& Miller, 1995). When comparing the average RT in a visual selection task with one resulting from a four-tofour response-to-stimulus mapping in an analog tactile task, as used here, it is obvious that RTs in the tactile task are significantly longer (whereas the error rates are also higher). This probably indicates that the tactile task is by far the more difficult one; this situation might be the result of people having less experience with tactile identification tasks as compared with visual tasks. The same observation also holds true for the comparison of auditory versus 
visual tasks. In addition, the differences in NP between Experiments 1 and 2 in the present study also show a pattern that fits with this interpretation, with greater NP in the more difficult experiment - though this difference did not reach statistical significance. Taking these results together, we can hypothesize that task difficulty might play a role in the selection difficulty of NP tasks, whether the stimuli are presented to the visual, auditory, or tactile modality, and that the size of NP effects represents a function of the difficulty of selection - which may well be independent of any modality-specific processes involved. This fits with the claim that NP is a phenomenon that - though it might work on different levels of stimulus representation (see Fox, 1995) - usually works on a central stimulus representation. One might state that this central stimulus representation is free of modality-specific information. This argument is in line with a cross-modal NP study by Buchner, Zabal, and Mayr (2003), who found comparable NP effects when visual and auditory primes and probes were intermixed. However, it should be noted that the assumption of a modality-free representation of the distractor is still quite speculative at this point; thus, further research that more directly compares the ignoring of distractors between visual and tactile tasks is needed before this interpretation can safely be stated.

In sum, the results of the two experiments reported here provide the first evidence concerning the active ignoring of distractor representations within the tactile modality. From this result, we conclude that tactile selection is achieved by an active ignoring of distractors, in addition to facilitation of target representations.

\section{AUTHOR NOTE}

Correspondence concerning this article should be addressed to C. Frings, Saarland University, Faculty of Behavioral Sciences, Department of Psychology, Building A2.4, P.O. Box 1511 50, D-66041 Saarbrücken, Germany (e-mail: c.frings@mx.uni-saarland.de).

\section{REFERENCES}

Allport, A. (1987). Selection for action: Some behavioral and neurophysiological considerations of attention and action. In H. Heuer \& A. F. Sanders (Eds.), Perspectives on perception and action (pp. 395-419). Hillsdale, NJ: Erlbaum.

AllPORT, A. (1993). Attention and control: Have we been asking the wrong questions? A critical review of twenty-five years. In D. E. Meyer \& S. Kornblum (Eds.), Attention and performance XIV: Synergies in experimental psychology, artificial intelligence, and cognitive neuroscience (pp. 183-218). Cambridge, MA: MIT Press.

Brungart, D. S., Simpson, B. D., Ericson, M. A., \& Scott, K. R. (2001). Informational and energetic masking effects in the perception of multiple simultaneous talkers. Journal of the Acoustical Society of America, 110, 2527-2538.

Buchner, A., Zabal, A., \& Mayr, S. (2003). Auditory, visual, and cross-modal negative priming. Psychonomic Bulletin \& Review, 10, 917-923.

Calvert, G. A., Spence, C., \& Stein, B. E. (Eds.) (2004). The handbook of multisensory processes. Cambridge, MA: MIT Press.

Craig, J. C. (1974). Vibrotactile difference thresholds for intensity and the effect of a masking stimulus. Perception \& Psychophysics, 15, 123-127.

CraIG, J. C. (1995). Vibrotactile masking: The role of response competition. Perception \& Psychophysics, 57, 1190-1200.

CRaIG, J. C. (2000). Processing of sequential tactile patterns: Effects of a neutral stimulus. Perception \& Psychophysics, 62, 596-606.
Craig, J. C., \& Evans, P. M. (1995). Tactile selective attention and temporal masking. Perception \& Psychophysics, 57, 511-518.

Dell'Acqua, R., Joliceeur, P., Sessa, P., \& Turatto, M. (2006). Attentional blink and selection in the tactile domain. European Journal of Cognitive Psychology, 18, 537-559.

Dell'Acqua, R., Turatto, M., \& Joliceur, P. (2001). Cross-modal attentional deficits in processing tactile stimulation. Perception \& Psychophysics, 63, 777-789.

Driver, J., \& Grossenbacher, P. G. (1996). Multimodal spatial constraints on tactile selective attention. In T. Inui \& J. L. McClelland (Eds.), Attention and performance XVI: Information integration in perception and communication (pp. 209-235). Cambridge, MA: MIT Press, Bradford Books.

Evans, P. M., \& Craig, J. C. (1991). Tactile attention and the perception of moving tactile stimuli. Perception \& Psychophysics, 49, 355-364.

Fox, E. (1995). Negative priming from ignored distractors in visual selection: A review. Psychonomic Bulletin \& Review, 2, 145-173.

FrINGS, C. (2006). Relevant distractors do not cause negative priming. Psychonomic Bulletin \& Review, 13, 322-327.

Frings, C., \& Wentura, D. (2006a). Negative priming is stronger for task-relevant dimensions: Evidence of flexibility in the selective ignoring of distractor information. Quarterly Journal of Experimental Psychology, 59, 683-693.

Frings, C., \& Wentura, D. (2006b). Strategy effects counteract distractor inhibition: Negative priming with constantly absent probe distractors. Journal of Experimental Psychology: Human Perception \& Performance, 32, 854-864.

FrIngs, C., \& WÜHR, P. (2007). Prime display offset modulates negative priming only for easy-selection tasks. Memory \& Cognition, 35, 504-513.

Hillstrom, A. P., Shapiro, K. L., \& Spence, C. (2002). Attentional limitations in processing sequentially presented vibrotactile targets. Perception \& Psychophysics, 64, 1068-1082.

Houghton, G., \& TipPeR, S. P. (1994). A model of inhibitory mechanisms in selective attention. In D. Dagenbach \& T. H. Carr (Eds.), Inhibitory processes in attention, memory, and language (pp. 53-112). San Diego: Academic Press.

Houghton, G., Tipper, S. P., Weaver, B., \& Shore, D. I. (1996). Inhibition and interference in selective attention: Some tests of a neural network model. Visual Cognition, 3, 119-164.

Johnston, W. A., \& Dark, V. J. (1986). Selective attention. Annual Review of Psychology, 37, 43-75.

Joordens, S., Betancourt, I., \& Spalek, T. M. (2006). Selective attention versus selection for action: Negative priming is not the result of distractors being unattended. Perception \& Psychophysics, 68, 890-896.

Kane, M. J., May, C. P., Hasher, L., Rahhal, T., \& Stoltzfus, E. R. (1997). Dual mechanisms of negative priming. Journal of Experimental Psychology: Human Perception \& Performance, 23, 632-650.

MacLeod, C. M., Chiappe, D. L., \& Fox, E. (2002). The crucial roles of stimulus matching and stimulus identity in negative priming. Psychonomic Bulletin \& Review, 9, 521-528.

MARKS, L. E. (2004). Cross-modal interactions in speeded classification. In G. A. Calvert, C. Spence, \& B. E. Stein (Eds.), The handbook of multisensory processes (pp. 85-105). Cambridge, MA: MIT Press.

May, C. P., KANE, M. J., \& Hasher, L. (1995). Determinants of negative priming. Psychological Bulletin, 118, 35-54.

MAYr, S., \& Buchner, A. (2006). Evidence for episodic retrieval of inadequate prime responses in auditory negative priming. Journal of Experimental Psychology: Human Perception \& Performance, 32, 932-943.

Milliken, B., Joordens, S., Merikle, P. M., \& Seiffert, A. E. (1998). Selective attention: A reevaluation of the implications of negative priming. Psychological Review, 105, 203-229.

NEILL, W. T. (1997). Episodic retrieval in negative priming and repetition priming. Journal of Experimental Psychology: Learning, Memory, \& Cognition, 23, 1291-1305.

Neumann, O. (1996). Theories of attention. In O. Neumann \& A. F. Sanders (Eds.), Handbook of perception and action: Vol. 3. Attention (pp. 389-446). San Diego: Academic Press.

Pashler, H. E. (1998). The psychology of attention. Cambridge, MA: MIT Press. 
Röder, B., Kusmierek, A., Spence, C., \& Schicke, T. (2007). Developmental vision determines the reference frame for the multisensory control of action. Proceedings of the National Academy of Sciences, 104, 4753-4758.

Rothermund, K., Wentura, D., \& De Houwer, J. (2005). Retrieval of incidental stimulus-response associations as a source of negative priming. Journal of Experimental Psychology: Learning, Memory, \& Cognition, 31, 482-495.

Ruthruff, E., \& Miller, J. (1995). Negative priming depends on ease of selection. Perception \& Psychophysics, 57, 715-723.

Simon, J. R. (1990). The effects of an irrelevant directional cue on human information processing. In R. W. Proctor \& T. G. Reeve (Eds.), Stimulus-response compatibility: An integrated perspective (pp. 31-86). Amsterdam: North-Holland.

Soto-Faraco, S., Ronald, A., \& Spence, C. (2004). Tactile selective attention and body posture: Assessing the multisensory contributions of vision and proprioception. Perception \& Psychophysics, 66, 1077-1094.

Soto-Faraco, S., Spence, C., Fairbank, K., Kingstone, A., HillSTROM, A. P., \& SHAPIRO, K. (2002). A crossmodal attentional blink between vision and touch. Psychonomic Bulletin \& Review, 9, 731-738.

Spence, C., \& Driver, J. (EdS.) (2004). Crossmodal space and crossmodal attention. Oxford: Oxford University Press.

Spence, C., Lloyd, D., McGlone, F., Nicholls, M. E. R., \& Driver, J. (2000). Inhibition of return is supramodal: A demonstration between all possible pairings of vision, touch, and audition. Experimental Brain Research, 134, 42-48.

Spence, C., Nicholls, M. E. R., Gillespie, N., \& Driver, J. (1998). Cross-modal links in exogenous covert spatial orienting between touch, audition, and vision. Perception \& Psychophysics, 60, 544-557.

TIPPER, S. P. (1985). The negative priming effect: Inhibitory priming by ignored objects. Quarterly Journal of Experimental Psychology, 37A, 571-590.
TIPPER, S. P. (2001). Does negative priming reflect inhibitory mechanisms? A review and integration of conflicting views. Quarterly Journal of Experimental Psychology, 54A, 321-343.

TuKEY, J. W. (1977). Exploratory data analysis. Reading, MA: AddisonWesley.

UtTAL, W. R. (1960). Inhibitory interaction of responses to electrical stimuli in the fingers. Journal of Comparative \& Physiological Psychology, 53, 47-51.

\section{NOTES}

1. One important question for future research will be to determine whether the masking effects seen in the tactile modality should be attributed to energetic masking (i.e., one of the vibrations becomes less perceptible) or to informational masking (i.e., it is hard to separate the vibrations), a topic discussed extensively for the auditory modality (see Brungart, Simpson, Ericson, \& Scott, 2001).

2 . We also analyzed whether prime response hand or prime target position interacted with any of the other factors. We found no influence of either prime response hand or prime target location on the NP effect (all $F \mathrm{~S}<1$ ). However, irrespective of the priming condition, probe reactions were an average of $172 \mathrm{msec}$ faster when the probe target and the prime target were presented to the same hand $[F(1,16)=37.47, p<.001$, $\left.\eta^{2}=.70\right]$. Moreover, we analyzed whether the tactile patterns differed with respect to NP effects. However, although the general identification times for the stimuli differed somewhat, NP was not modulated by these differences $(F<1)$.

3. As in Experiment 1, NP was not significantly modulated by prime hand, prime position, or stimulus type (all $F \mathrm{~s}<1$ ).

(Manuscript received June 9, 2007; revision accepted for publication November 5, 2007.) 